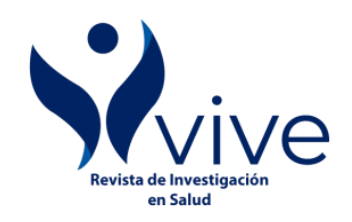

Revista de Salud VIVE. Revista de Investigación en Salud https://doi.org/10.33996/revistavive.v3i7.41 Volumen 3 | No. 7 | Enero - Abril 2020 http://revistavive.org ISSN: $2664-3243$

\section{Conductas de riesgo en adolescentes estudiantes de nivel secundario}

\author{
Conduct of risk in adolescents secondary level students \\ Comportamentos de risco em adolescentes estudantes do \\ ensino médio
}

Nicacia Romero Mamani romeronicacia6@gmail.com

Recibido septiembre 2019 / Revisión octubre 2019 / Aceptado 1 de enero 2020

\section{RESUMEN}

Introducción: Las investigaciones en adolescentes puedan ser de gran utilidad para plantear, con evidencia, la necesidad de realizar actividades preventivas enfocadas a conductas de riesgo. Objetivo: Determinar las conductas de riesgo más prevalentes y su relación con edad y sexo de los de los adolescentes estudiantes de la Ciudad de Villazón. Materiales y métodos: El tipo de investigación es epidemiológico, observacional, descriptivo, analítico y transversal. Los resultados obtenidos plantean que existe relación entre el género y las conductas de riesgo como, el consumo de alcohol en mayores de 18 años y en menores de 18 años; consumo de tabaco los hombres muestran 8 veces mayor riesgo que las mujeres; también los hombres mostraron 5 veces mayor riesgo de consumo de drogas que las mujeres; además de no usar condón en su primera relación sexual y los hombres mostraron mayor riesgo de tener enfermedades de transmisión sexual que las mujeres. Conclusión: Las conductas de riesgo más prevalentes en adolescentes, son el consumo de tabaco, consumo de drogas ilícitas y las relaciones sexuales.

Palabras clave: conductas; riesgo; adolescentes; relaciones sexuales

\section{ABSTRACT}

Introduction: Investigations in adolescents can be very useful to present, with evidenced, the needs for preventive activities focused on risk behaviors. Therefore, the objective was to determine the most prevalent risk behaviors of adolescents and their relationship with age and sex of the students of the City of Villazón. Materials and methods: The type of research is epidemiological, observational, descriptive, analytical and transversal. The results obtained suggest that there is a relationship between gender and risk behaviors such as alcohol consumption in people over 18 years of age and in children under 18; tobacco use men show 8 times greater risk than women; men also showed 5 times greater risk of drug use than women; in addition to not using a condom in their first sexual intercourse and men showed a higher risk of having sexually transmitted diseases than women. Conclusion: the most prevalent risk behaviors in adolescents are tobacco use, illicit drug use and sexual relations.

Key words: behaviors; risk; adolescents; sexual relations
NR: Universidad San Francisco Xavier de Chuquisaca, Bolivia. 
NR: Universidad San Francisco Xavier de Chuquisaca, Bolivia.

\section{RESUMO}

Introdução: As investigações em adolescentes podem ser muito úteis para apresentar, com evidências, a necessidade de atividades preventivas focadas em comportamentos de risco. Portanto, o objetivo foi determinar os comportamentos de risco mais prevalentes dos adolescentes e sua relação com a idade e o sexo dos estudantes da cidade de Villazón 2017. Materiais e métodos: O tipo de pesquisa é epidemiológica, observacional, descritiva, analítica e transversal. Os resultados obtidos sugerem que existe uma relação entre gênero e comportamentos de risco, como o consumo de álcool em pessoas acima de 18 anos e em menores de 18 anos; homens que usam tabaco apresentam risco 8 vezes maior que mulheres; os homens também apresentaram risco 5 vezes maior de usar drogas do que as mulheres; além de não usar camisinha na primeira relação sexual, os homens apresentaram maior risco de ter doenças sexualmente transmissíveis do que as mulheres. Conclusão: os comportamentos de risco mais prevalentes em adolescentes são uso de tabaco, uso de drogas ilícitas e relações sexuais.

Palavras-chave: comportamentos; risco; adolescentes; relação sexual

\section{INTRODUCCIÓN}

$\mathrm{U}$ n sector de la población con mayor vulnerabilidad de adquirir conductas de riesgo que afecten la salud son los adolescentes, debido que en esta etapa de la vida ocurre un proceso creciente de maduración física, psicológica y social que lleva al ser humano a transformarse en un adulto. En este período, ocurren cambios rápidos y de gran magnitud, la persona se hace tanto biológica, como psicológica y socialmente madura y capaz de vivir en forma independiente (1). Las conductas de riesgo (CR) en los adolescentes se definen como aquellas actividades que incrementan las posibilidades de deteriorar el estado de la salud por morbilidad o mortalidad. Existen 6 categorías de CR prioritarias según el Centro de Control y Prevención de Enfermedades de Estados Unidos de Norteamérica (CDC): uso de tabaco; consumo de alcohol y otras drogas; comportamientos sexuales que contribuyen a embarazos no deseados y a infecciones de transmisión sexual; comportamientos alimentarios no saludables;

sedentarismo; comportamientos que favorezcan accidentes y violencia (2).

En mayo de 2014 en Ginebra/Washington el informe sobre salud para los adolescentes del mundo de la Organización Mundial de la Salud (OMS) revela que la violencia interpersonal, las heridas por siniestros viales y el suicidio son las tres principales causas de muerte entre los adolescentes de 10 a 19 años de las Américas (3). La depresión es una de las tres primeras causas de enfermedad y discapacidad en este grupo en la región. El documento muestra que brindar más atención a esos problemas específicos mejoraría la salud de la población a lo largo de su vida, en particular si se abordan las condiciones y los comportamientos que se inician o se refuerzan en la adolescencia (3).

La Organización Mundial de la Salud (OMS) en una nota descriptiva declara que aproximadamente uno de cada seis habitantes del mundo es un adolescente, lo que significa que 1200 millones de personas tienen entre 10 y 19 años. 
La mortalidad y la morbilidad entre los adolescentes son estadísticamente elevadas. Las enfermedades pueden afectar a la capacidad de los adolescentes para crecer y desarrollarse plenamente. El consumo de alcohol o tabaco, la falta de actividad física, las relaciones sexuales sin protección y/o la exposición a la violencia pueden poner en peligro no solo su salud actual, sino también la de años posteriores (4). Fomentar prácticas saludables durante la adolescencia y adoptar medidas para proteger mejor a los jóvenes contra los riesgos sanitarios es fundamental para la prevención de problemas de salud en la edad adulta y para la futura infraestructura de salud y social de los países (4).

Las tendencias demográficas indican que, en 2015, había en América Latina y el Caribe 107 millones de niñas y adolescentes entre 0 y 19 años, cifra que corresponde al $17 \%$ de la población total de la región. Esta proporción es similar, aunque levemente menor a la de los varones del mismo grupo de edad, quienes representan el $18 \%$ de la población de la región (5).

Del total de la población entre 0 y 19 años de edad en América Latina, 11\% corresponden a niñas y adolescentes que habitan en zonas rurales y $38 \%$, a niñas y adolescentes que viven en zonas urbanas (5).

En Bolivia se realizó en la ciudad de La Paz en el año 2004, un estudio en el que se evidencia solo la conducta de las relaciones sexuales y la asociación con el consumo de alcohol, el resultado en esta investigación mostró las conductas de alto riesgo, una adolescente que consume bebidas alcohólicas tiene 5 a 7 veces más probabilidades de iniciar su actividad sexual que otra que no está expuesta a este hábito registrándose $21,5 \%$ de consumo de alcohol (6).
Por todo el análisis realizando en la literatura, se pretende mediante el estudio determinar la prevalencia de las conductas de riesgo del adolescente y su relación con la edad y sexo en estudiantes de nivel secundario de la Ciudad de Villazón.

Se maneja como hipótesis que las conductas de riesgo de mayor prevalencia, es el consumo de alcohol, tabaco y las relaciones sexuales en los adolescentes en estudiantes de la Ciudad de Villazón.

La Organización Mundial de la Salud define a la adolescencia como el período comprendido entre los 10 y 19 años de edad (1). La adolescencia es aquella etapa del desarrollo ubicada entre la infancia y la adultez, en la que ocurre un proceso creciente de maduración física, psicológica $\mathrm{y}$ social que lleva al ser humano a transformarse en un adulto. En este período, en el que ocurren cambios rápidos y de gran magnitud, la persona se hace tanto biológica, como psicológica y socialmente madura y capaz de vivir en forma independiente. Las características del desarrollo psicosocial normal en la adolescencia son el resultado de la interacción entre el desarrollo alcanzado en las etapas previas del ciclo vital, factores biológicos inherentes a esta etapa el desarrollo puberal y el desarrollo cerebral propio de este período, fenómeno a la vez relacionado en parte con los cambios hormonales de la pubertad y la influencia de múltiples determinantes sociales y culturales (7).

Desde la década del 90 se analiza la influencia de las conductas de riesgo en la salud integral de los adolescentes; entre los que se encuentran; los personales: su historia personal; sus modos de resolver situaciones infantiles de conflicto; su programa neurobiológico; la utilización de determinados mecanismos habituales de defensa frente a situaciones desestabilizadoras de su identidad, entre 
otros. Los precipitantes: La percepción subjetiva del impacto de las experiencias que le toca vivir; la capacidad de afrontamiento con que se maneja; el grado de capacidad y flexibilidad cognitiva para la resolución de problemas. Los de contexto: la existencia o no de redes de apoyo solidario con que cuente; el nivel de integración, la capacidad para diferenciar lo real de lo imaginario y para procesar la información de manera coherente (8).

La interacción de todos estos aspectos va a determinar la peculiar manera en que cada adolescente resolverá qué grado de riesgos asumirá en sus respuestas adaptativas (8).

El consumo de alcohol incrementa el riesgo de provocar una amplia variedad de problemas sociales de una manera dosis dependiente, sin que existan evidencias de un efecto umbral. En general, cuanto más grave resulte el delito o la lesión, tanto más probable que el consumo de alcohol haya sido el causante (9).

En cuanto al cigarrillo según la OMS (2006), el tipo de consumo está asociado con el número de cigarrillos consumidos al día. Así de acuerdo con la OMS (2003) los fumadores se clasifican en leves, moderados y severos en la siguiente escala: fumador leve, consume menos de 5 cigarrillos diarios; fumador moderado, fuma un promedio de 6 a 15 cigarrillos diarios; fumador severo, fuma más de 16 cigarrillos por día en promedio (10).

El fenómeno de las drogas ha experimentado en los últimos años un profundo proceso de transformación, en paralelo a la evolución de las sociedades desarrolladas. La heroína ha sido sustituida por sustancias como el cannabis, la cocaína o el éxtasis, combinadas entre sí o mezcladas con alcohol y tabaco. Este cambio de las sustancias también se ha acompañado de nuevos patrones de consumo. Estamos ante consumidores cada vez más jóvenes, perfectamente integrados en la sociedad, que consumen con fines recreativos y socializantes y para quienes estos consumos son actos triviales cuyos riesgos desdeñan (11).

Ampliamente en numerosas publicaciones científicas. Existe evidencia científica para su uso terapéutico en el caso de las náuseas y vómitos secundarios al tratamiento con antineoplásicos, la pérdida de apetito en VIH, cáncer terminal y el tratamiento del dolor neuropático en la esclerosis múltiple. (11).

Todo consumo implica un riesgo, pero el de cannabis es mayor en la adolescencia: provoca cambios neurobiológicos durante el periodo de maduración cerebral. El inicio temprano del consumo se asocia con la aparición de trastornos mentales en la edad adulta (11).

\section{MATERIALES Y METÒDOS}

$\mathrm{E}$ sta fue una investigación epidemiológica, descriptiva, con un componente analítico, transversal, con las variables dependientes (consumo de cigarrillo de tabaco, bebidas alcohólicas, drogas, enfermedades de trasmisión sexual y las relaciones sexuales). La población está conformada por 629 estudiantes de sexto de secundaria de los cuales se toma como objeto de investigación a los estudiantes adolescentes de 15 a 19 años de edad. El tamaño de muestra fue representativo de 473 estudiantes de las nueve unidades educativas de la ciudad de Villazón. El método utilizado para la selección de la muestra fue a través del método no probabilístico con un muestreo intencional o de conveniencia. Se siguieron los lineamientos éticos de toda investigación. Se utilizó como base la encuesta sobre comportamientos de riesgo, propuesto por el Banco Interamericano de Desarrollo-División de Protección Social y Salud. 


\section{RESULTADOS Y DISCUSIÓN}

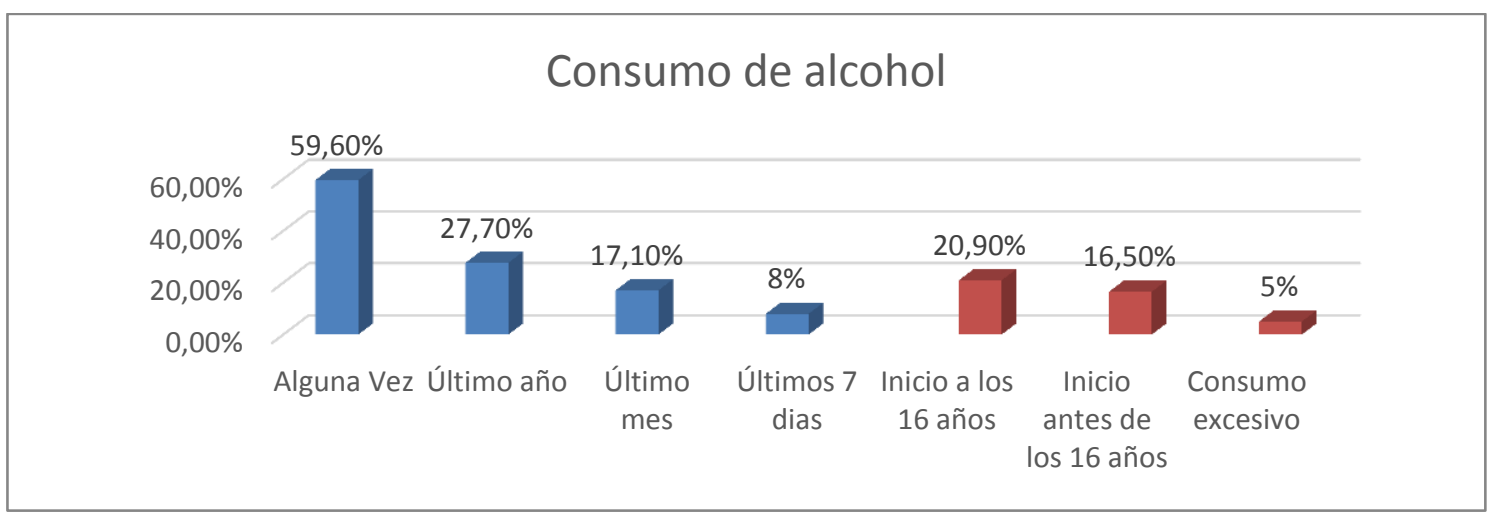

Gráfico 1. Consumo de alcohol en adolescentes de sexto secundaria de la Ciudad de Villazón

\section{Consumo de tabaco en adolescentes}

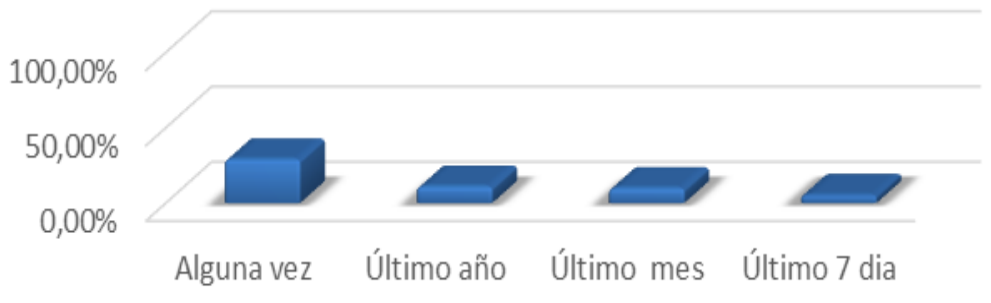

Gráfico 2. Consumo de tabaco en adolescentes de sexto secundaria de la Ciudad de Villazón.

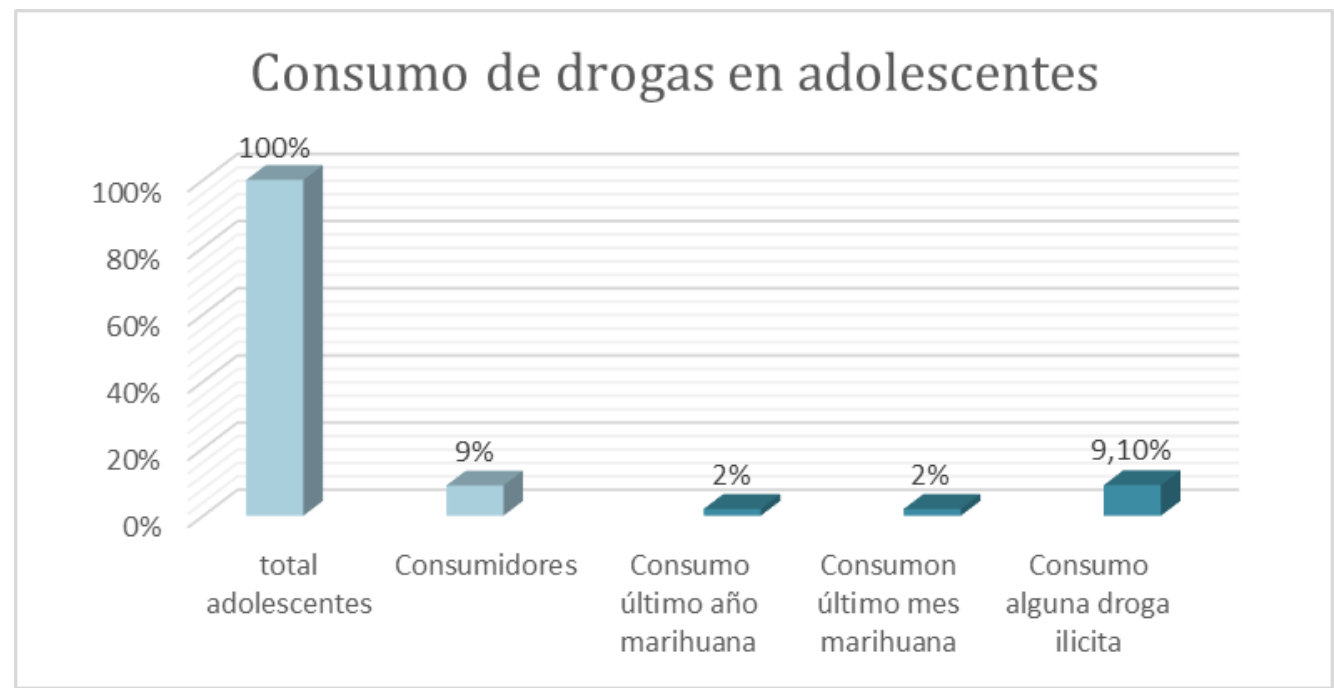

Gráfico 3. Consumo de drogas ilícitas en adolescentes de sexto secundaria de la Ciudad de Villazón. 


\section{DISCUSIÓN}

A nte las conductas de riesgo en adolescentes, es importante considerar los factores $y$ conductas protectoras, la mayoría de ellos espejos de las de riesgo, agrega un componente importantísimo para aumentar la eficacia del trabajo preventivo. Se entiende como conductas de protección aquellas acciones voluntarias o involuntarias, que pueden llevar a consecuencias protectoras para la salud, son múltiples y pueden ser bio- psicosociales (12). Existen niños y adolescentes que, a pesar de estar expuestos a condiciones de vida adversas, llegan a ser adultos saludables y positivos lo que apoya la teoría de la resiliencia. Entendida como aquella característica humana que permite recuperarse y superar la adversidad. Es difícil saber si un adolescente relativamente no desafiado por el ambiente la está desarrollando, y porque estar libre de riesgos no significa necesariamente estar preparado para la adultez, es importante poder identificar fortalezas universales que pueden ser útiles a todos los adolescentes (12).

Los adultos más cercanos, como son los padres, constituirán una fuente fundamental de modelaje, pero también pueden ser factores externos positivos, al ser fuentes de apoyo y crecimiento, los pares, el colegio, la comunidad y la sociedad (12).

Si bien algunas características de la adolescencia facilitarían la realización de conductas saludables, existen multitud de factores que favorecen su iniciación en los contextos de riesgo. Por ejemplo, más allá de poseer una capacidad cognitiva que permita evaluar riesgos y beneficios de las conductas, variables como la búsqueda de sensaciones, la baja percepción de riesgo o la importancia de la aceptación de los pares, favorecen que una buena parte de los adolescentes lleven a cabo conductas de riesgo (13).

Las conductas de riesgo (consumo de alcohol, tabaco y otras drogas, mala alimentación, sedentarismo y sexualidad de riesgo), entre adolescentes (2).

En este estudio los adolescentes bebieron alguna vez en la vida $66.2 \%$ en hombre y $53.3 \%$ las mujeres (OR=1.7, IC95\% $(1.1,2.4)$, en los últimos 7 días un 9.5\% para los hombres y para las mujeres $6.6 \%$, consumiendo los mayores de 18 años en un $69.4 \%$ (OR=1.7, IC95\% (1.1, 2.4). Para la conducta de consumo de tabaco los adolescentes en el caso de este estudio se observa que la distribución es por encima en adolescentes que consumen tabaco alguna vez en la vida $59.8 \%$ en el último año $22.4 \%$, en el último mes $34.4 \%$ y en los últimos 7 días $16.6 \%$.

Para la conducta de drogas en los adolescentes manifestaron haber probado la marihuana como la principal droga. En este este estudio la distribución de drogas ilícitas es de $9 \%$ y para la marihuana $4 \%$

Este estudio busca conductas de riesgo en relación con el género y edad de los adolescentes de sexto de secundaria en la Ciudad de Villazón, se observa asociaciones significativas que permiten afirmar que las conductas de riesgo más frecuente es el consumo de tabaco, consumo de drogas principalmente la marihuana, asociado a la conducta de relaciones sexuales. Para los adolescentes hombres tienen de 3 a 8 veces mayor riesgo de consumir tabaco en relación a las mujeres y la edad de inicio es a los 15,8 años.

Para abordar esta problemática se debe incorporar elementos como los factores y conductas de protección desde el punto de vista del adolescente como del padre de familia.

Los hallazgos de este estudio son relevantes y cabría tenerlos en cuenta en la 
medida en que alerte sobre la situación de las conductas de riesgo entre los adolescentes de la Ciudad de Villazón, en las conductas, arriba mencionados; así estos resultados muestran la necesidad de proponer una estrategia de intervención de promoción y prevención, adaptados a los adolescentes, para prevenir aquellas conductas de riesgo que dificulten su bienestar a corto, mediano y largo plazo.

\section{CONCLUSIÓN}

$\mathrm{N}$ o se muestran diferencias significativas en relación con la edad en la conducta de riesgo consumo de alcohol. En relación al consumo de tabaco por edad no se encontraron diferencias. En relación al consumo de drogas ilícitas por edad no se encontraron diferencias. Acerca de la variable relaciones sexuales y edad no se encontraron diferencias significativas. En comparación de la conducta de enfermedades de transmisión sexual y edad no se tiene diferencias significativas.

Se recomienda al personal docente de las unidades educativas transversalizar los temas de conductas de riesgo del adolescente y coordinar con el personal de salud, en detección de riesgos y realización de ferias educativas con participación de los adolescentes.

- Conflicto de intereses. Ninguno declarado por la autora.

- Financiación. Ninguna declarada por la autora.

- Agradecimiento: Ninguno manifestado por la autora.

- Investigación realizada considerando los tratados bioéticos.

\section{REFERENCIAS BIBLIOGRÁFICAS}

1. Organización Mundial de la salud. Desarrollo en la adolescencia. Programas y proyectos. Salud de la madre, el recién nacido, del niño y del adolescente. [Sitio en internet]. Consultado el 16 junio de 2018. Disponible en: http://www.who.int/maternal_child_ad olescent/topics/adolescence/dev/es/

2. Díaz F, Gaete V. Conductas de riesgo en adolescentes con patología crónica compleja bajo control en un policlínico de un hospital pediátrico de Santiago. Rev. Med. Chile. 2016;114,734-742

3. Organización Mundial de la Salud Estrategia mundial para la salud de la mujer, el niño y el adolescente 20162030: Sobrevivir, Transformar, Prosperar. [Sitio en internet] [Acceso el 18 de enero de 2017]. Disponible en:

http://www.who.int/maternal_child_a dolescent/documents/estrategiamundial-mujer-nino-adolescente2016-2030.pdf

4. Glenn T, Linn L. La OPS/OMS llama a que se preste mayor atención a la salud de los adolescentes para evitar muertes y mejorar su salud. [Sitio en internet]. Washintong, D.C.: Oficina regional para las Américas de la Organización Mundial de la Salud. acceso 17 de enero 2017]. Disponible en:http://www.paho.org/hq/index.ph p?option $=$ com_content\&view $=$ article $\&$ $\mathrm{id}=9579 \% 3 \mathrm{~A} 2014$ -

pahowhoadolescents-health-preventdeathsimprovehealth\&Itemid=1926\&l ang=es

5. Céspedes C, Robles C. Niñas y adolescentes en América Latina $\mathrm{Y}$ el caribe: Deudas de igualdad. [Sitio en internet acceso el 16 de enero 2017]. Disponible en: https://www.unicef.org/ecuador/Nin as_y_Adolescentes_en_America_Latina _y_el_Caribe

6. Torrico A, Salas A, Gutiérrez A, Arce M, Salazar J. Factores de riesgo asociados al inicio de relaciones sexuales en adolescentes mujeres estudiantes de secundaria en La Paz, Bolivia. Revista Sociedad Boliviana de Pediatría. 2004; 43, (1): 3-7 
7. Gaete V. Desarrollo psicosocial del adolescente. Rev. Chil. Pediatr. 2015; 86(6): 436-443

8. Pineda S, Aliño M. Manual de prácticas para la atención integral a la salud en la adolescencia. Cuba: MINSAP; 2002. Consultado 23 de mayo de 2018. Disponible en: http://www.sld.cu/galerias/pdf/sitio s/prevemi/manual_de_practicas_clinic as_para_la_atencion_integral_a_la_salu d_de_los_adolescentes.pdf

9. Anderson P, Gual A, Colon J. Alcohol y atención primaria de la salud. Informaciones clínicas básicas para la identificación y el manejo de riesgos y problemas. Washington. D.C. 2008. P2-9
10. Londoño C, Rodriguez I, Gantiva CA. Cuestionario para la clasificación de consumidores de cigarrillo (C4) para jóvenes. Rev. Perspect. Psicol.2011; 7, (2): 281-291

11. Ministerio de Salud y Consumo, Soria B. Una guía útil sobre drogas. 2007.

12. Corona F, Peralta E. prevención de conductas de riesgo. Rev. Med. Clin. Condes. 2011; 22, (1): 68-75

13. Castillo LC, Álvarez A, Bañuelos Y, Valle $M$, Valdez $C$, et al. Edad, género y resiliencia en la conducta sexual de riesgo para ITS en adolescentes al Sur de México. Enfermería Global. 2017; 45: $168-177$ 